\title{
Una tragedia canadiense
}

\author{
Paul G. BahN ${ }^{\star}$, Robert G. Bednarik ${ }^{\star \star}$ y Jack Steinbring ${ }^{\star \star *}$
}

La publicación del artículo firmado por Isabel García Fernández (Revista de Arqueología 174, octubre 1995) con el título "The Teaching Rocks", nos ha producido una profunda consternación al presentarse como un informe ingenuo y completamente falto de crítica, acerca de una estructura que consideramos ha sido uno de los mayores desastres para el arte rupestre del Canadá. Lo que la autora describe repetidamente como una "estructura protectora» debería clasificarse más exactamente como "estructura destructora". Nos preguntamos si Isabel García Fernández realmente visitó el sitio de Peterborough; y si así lo hizo, estamos asombrados de que no haya dado noticia del daño que esta estructura ha causado al conjunto, así como de su falta de mantenimiento. EI texto está claramente inspirado por entero en la literatura "oficial» que, acerca de la estación proporciona el Instituto de Conservación Canadiense ( $\mathrm{CCl}$, Canadian Conservation Institute) - que podría calificarse casi de propaganda-, el cual insiste en presentar al mundo este proyecto como un epítome de la conservación del arte rupestre, aunque nunca haya publicado una palabra sobre el considerable daño que la misma ha originado. Tenemos, por tanto, que artículos tales como el de Isabel García Fernández, no sólo presenten un cuadro falso, sino que puedan además llevar a los lectores interesados a creer que la cubrición de manifestaciones de arte rupestre dentro de este tipo de estructuras es una buena idea que debiera llevarse a cabo en otros lugares.

La estructura, vista tanto desde el exterior, como en fotografías, muestra un aspecto impresionante y sorprendente, sin embargo, una observa-

\footnotetext{
* England. Vice-president, AURA (Australian Rock Art Research Association).

** Australia. Convenor, IFRAO (International Federation of Rock Art Organizations).

*** USA. Professor, Ripon College.
} 

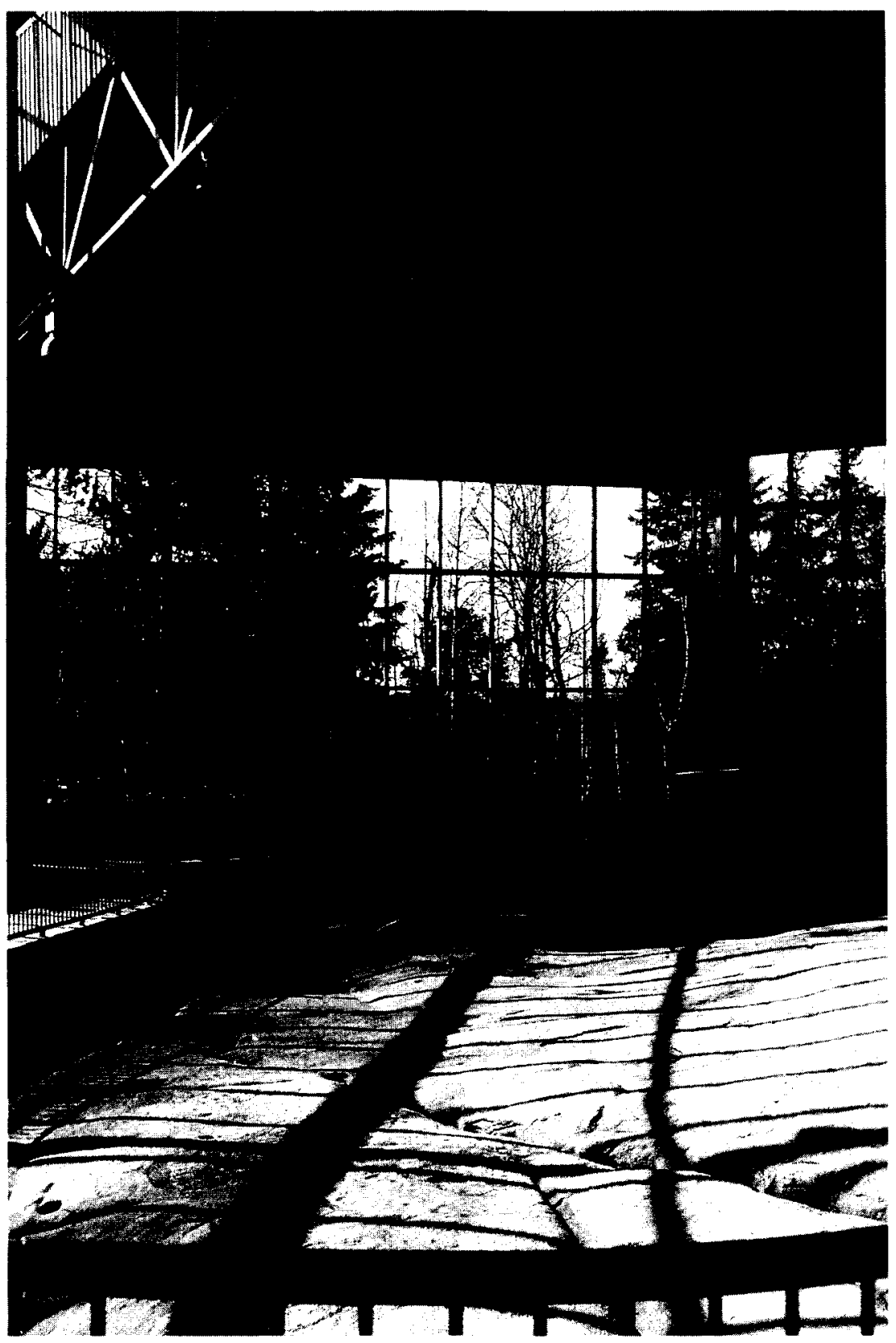

Figura 1. Interior de la estructura de Peterborough, mostrando algunos ventanales dañados y su condensación. 
ción más atenta de la evolución y actual estado del proyecto, pone de manifiesto algunos aspectos inquietantes. No sólo hubo una ausencia de objetivos claros en la planificación, sino que la construcción de la estructura - que costó casi 800.000 dolares canadienses de la época- probablemente no hubiera sido necesaria en absoluto. $\mathrm{El} \mathrm{CCl}$, requerido por el Ministerio de Recursos Naturales de Ontario en 1980, concluyó que la exposición al hielo, así como el crecimiento de varias especies de algas, habían contribuido a un deterioro significativo del sitio, recomendando que la roca principal fuese encerrada en una estructura protectora para eliminar así la acción de la lluvia, nieve y cursos de agua superficiales, y con ello suprimir el crecimiento de las algas. También se señaló al vandalismo como uno de los peligros evidentes pese a que el vallado ya existente en este remoto sitio hubiera servido para eliminar dicho factor (de cualquier modo, si ningún sitio de arte rupestre está completamente a salvo del vandalismo, ¿por qué, en un sitio remoto y mínimamente dañado por esta causa, se ha considerado vital para su protección una cubierta artificial?).

Resulta muy dificil creer que la decisión de edificar esta estructura fuera tomada únicamente después de descartar las restantes posibilidades, tal y como afirma el $\mathrm{CCl}$. Habría resultado mucho más sencillo y barato arrancar aquellos árboles cuya sombra favorecía la aparición de las algas (que en este clima crecen muy lentamente), y cubrir la roca cada invierno de modo que se la preservase de daños causados por nevadas o hielos. La primera impresión es que la decisión tomada optó de forma deliberada por una solución pretenciosa, de alta tecnología y de alto coste, con el objetivo de exportar una imagen firme y contundente del compromiso de Canadá con la conservación del arte rupestre.

Por desgracia, la propia estructura ha dañado el sitio de una manera irreparable. Por ejemplo, toda la estructura de cemento, metal y cristal, descansa directamente sobre la roca decorada, pese a que increíblemente, no se ha publicado nunca nada acerca de la capacidad del sitio para soportar el peso de tal construcción sin efectos perjudiciales, ni tampoco existe información acerca de la carga ejercida sobre la superficie decorada o del contexto geofísico. Es muy probable que el peso de la estructura haya sido un factor determinante a tener en cuenta en relación a la significativa amplitud y profundidad de una grieta situada en el extremo NE de la estación, que se ha hecho mucho más evidente en estos dos últimos años.

De la misma manera, no se han proporcionado nunca detalles de cómo se ensambló la estructura a la roca, o de cómo se efectuó la impermeabilización y en qué consistió la misma. A juzgar por la considerable filtración 
de agua y la cantidad de hielo, que ha pasado a convertirse en un elemento permanente, visible en el extremo NE de la roca desde Marzo de 1995, parece que el fracaso ha sido absoluto y el resultado catastrófico.

Durante la construcción de la estructura in situ, el conjunto fue protegido con una capa de fardos de paja cubiertos por placas de madera de contrachapado recubiertas a su vez por placas de plástico. Sin embargo, los fardos de paja se prendieron por las chispas procedentes de los soldadores. Uno de estos fuegos fue el causante de una gran mancha blanca debida al uso de extintores y a los efectos térmicos sobre la roca soporte, todavía visible sobre 8 ó 9 petroglifos, en la parte Este del sitio.

El informe interno del $\mathrm{CCl}$, no publicado, afirma que «no se observaron daños en la superficie de la roca en forma de arañazos $u$ otras marcas excepto en las áreas donde los árboles fueron arrancados con material pesado". Con todo, pueden observarse sobre los petroglifos no impermeabilizados en el extremo NE de la roca, una buena cantidad de grandes y significativos arañazos causados por las cadenas de una de las retroexcavadoras.

Los visitantes, especialmente en verano, encuentran la estructura insuficientemente ventilada, con falta de aire fresco; y sorprendentemente sin ningún tipo de control de los niveles de dióxido de carbono presentes en la estructura, factor de importancia en la conservación de la superficie de la roca. Los lectores automáticos existentes toman constantemente la humedad y temperatura en el techo y sobre la roca, pero el control de los mismos por el $\mathrm{CCl}$ es poco frecuente, por no decir mínimo.

Los muros de cemento que soportan la estructura están agrietados en numerosos puntos y las sales lixiviadas así como la humedad consiguen penetrar a través de los mismos. Gran parte de la estructura está diseñada en unos grandes ventanales con dobles paneles de vidrio, sin embargo, éstos no sólo no han sido limpiados nunca en su parte interior o exterior, sino que al menos trece de las láminas de vidrio externas han quedado selladas y la condensación ha penetrado entre los paneles de vidrio, permaneciendo algunos en esta condición desde hace por lo menos dos años. Por las mañanas pueden apreciarse zonas de condensación sobre la roca y restos de pequeños charcos en los huecos de la superficie decorada. EI agua se filtra por las goteras del techo, provocando una importante oxidación de las piezas metálicas y la pintura está desconchándose y cayendo sobre la superficie grabada. Las rocas permanecen polvorientas en todo momento -en ausencia de un lavado natural por la lluvia- y el personal de mantenimiento se ve obligado a limpiarlas con cepillos blandos y con aspiradora. 


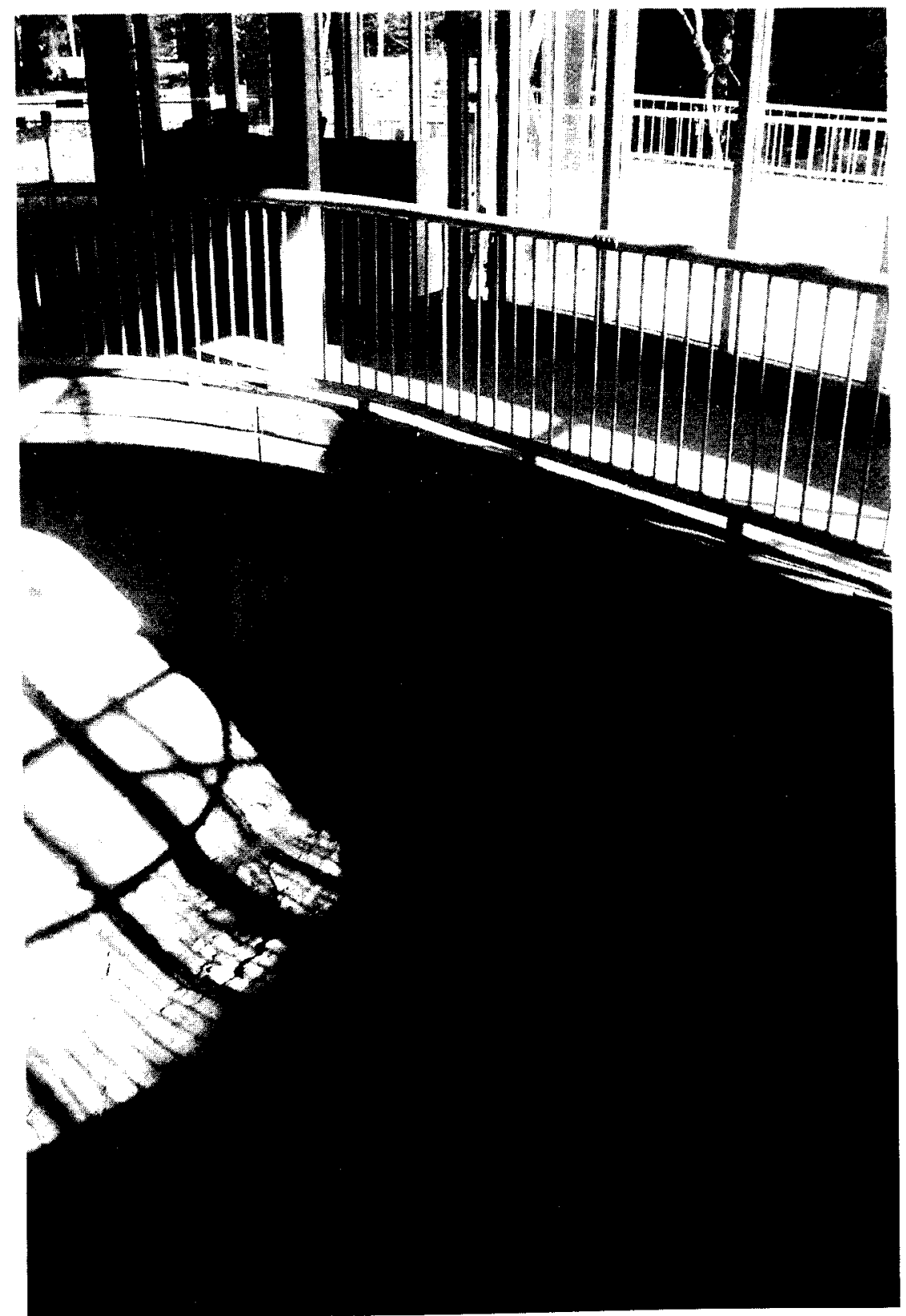

Figura 2. Extremo noroeste de la superficie de petroglifos donde puede observarse la existencia de un gran número de arañazos realizados por la retroexcavadora, y el agua/hielo que la inunda. 
En resumen, tan sólo diez años después de su instalación, la estructura presenta unas condiciones paupérrimas, existiendo una amenaza creciente para las manifestaciones artísticas. En términos de museología, la situación es incluso peor: se construyó un buen centro de visita de 2.286 $\mathrm{m}^{2}$ hace 6 años, a 385 metros del sitio principal de petroglifos, para proporcionar al público información sobre la cultura «algonkina» y sobre el arte rupestre antes de llegar al sitio.

Este edificio, con una sala para la proyección de la película "The Teaching Rocks", que cuenta además con cafetería, tienda de regalos y otros servicios, permanece totalmente vacío, por lo que los visitantes deben confiar por completo para su información en un personal de atención que a veces se encuentra en dificultades, y en unos escuetos folletos explicativos.

Inevitablemente, la estructura de Peterborough, es una intrusión en el medioambiente del sitio. Aislar la roca del mundo exterior la ha llevado completamente a la «muerte» y la ha convertido en una pieza de museo.

Resulta escandaloso que, tras el daño causado al arte rupestre por la construcción de esta estructura y el consiguiente deterioro de las condiciones de conservación, el $\mathrm{CCl}$-a pesar de su nombre-continue proponiendo más actuaciones perjudiciales para los petroglifos. Isabel García Fernández, en un pie de nota, señala de forma inocente que: «Recientemente se han realizado análisis, dirigidos por el Instituto Canadiense de Conservación, para determinar el mejor modo de limpiar los petroglifos y conseguir restaurar su estado original». Lo que esta acción podría acarrear en última instancia es la propia destrucción del arte. El CCl lamentó el hecho de que muchos de los grabados hubieran sido previamente coloreados con lápiz negro indeleble. Si bien es cierto que esta solución resultó efectivamente poco afortunada, no lo es menos que ahora hemos de convivir con esta situación de forma inevitable. La pintura consigue que los grabados coloreados sean vistos más facilmente por el público (una consideración importante para un sitio que ha sido orientado como atracción turística), mientras que aquellos sin pintura -bien en el interior, bien en el exterior de la estructura- resultan prácticamente invisibles para un ojo no experimentado.

Aún así el $\mathrm{CCl}$ ha llevado a cabo experimentos en la limpieza de tres de los grabados en el interior de la estructura, con el objetivo de aclarar el color en contraposición al fondo oscuro que presenta el contexto sobre el que están expuestos, tratando de restituirlos a su estado original. Sus técnicos han decidido que la mejor técnica es la abrasión por aire seco, utilizando cuentas de cristal o cristal machacado impulsado por nitrógeno, 


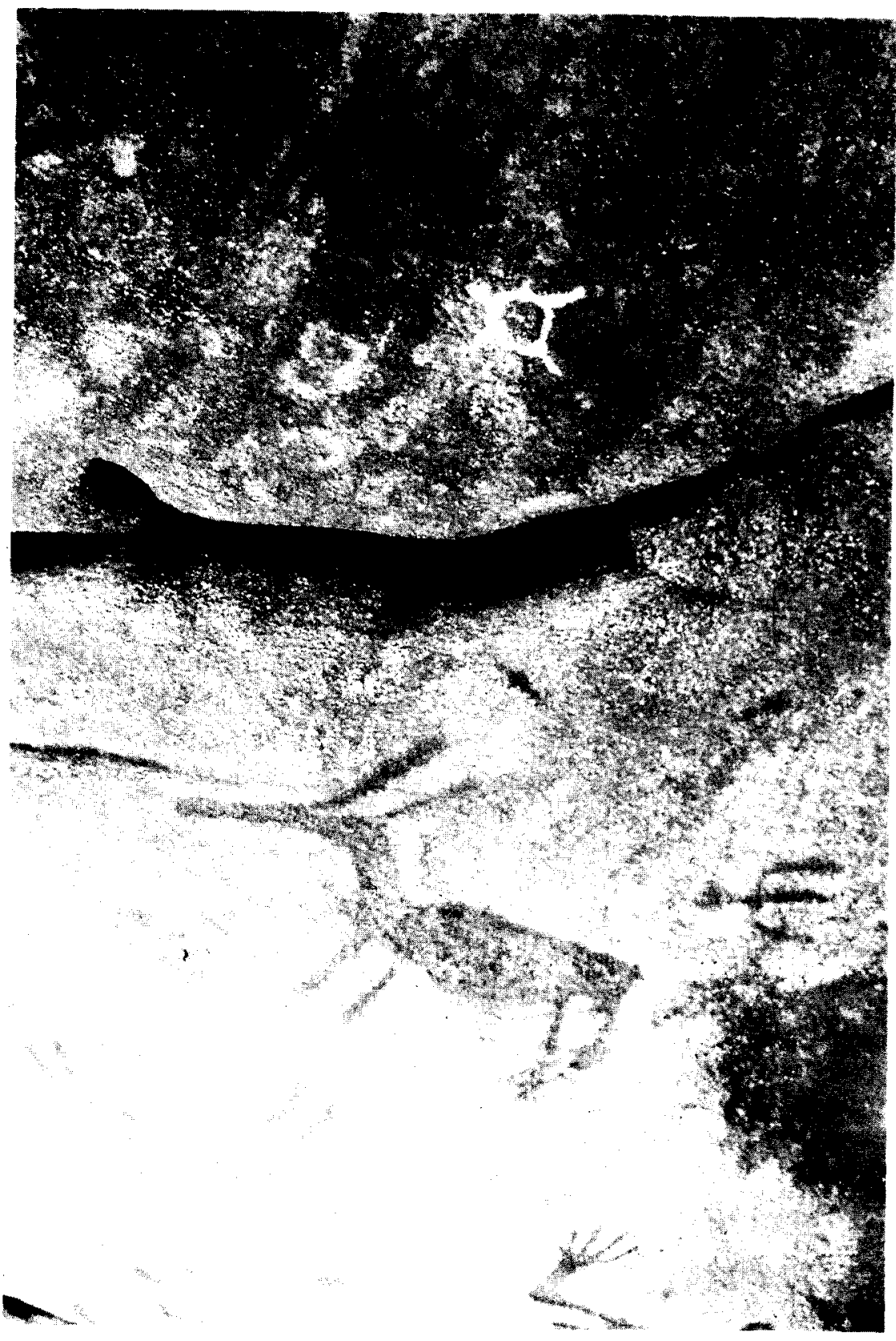

Figura 3. Petroglifo de una tortuga, "abrasionado" por el $\mathrm{CCl}$, evidenciando de forma clara la diferencia radical con los motivos pintados. 
aunque esto en ocasiones produzca el desprendimiento de granos de la roca. Encuentran esto tolerable, puesto que la pérdida es menor que aquella producida por la exposición al hielo, aunque hay una diferencia entre el daño natural producido al sitio y el daño causado por el hombre e inflingido por razones o motivos no explícitos. La figura de una pequeña tortuga sobre el fondo oscuro ha sido "limpiada" de tal modo que ahora destaca por su extrema claridad. Por ello el proyecto, —defendido aún por el $\mathrm{CCl}$, aunque afortunadamente, no ejecutado todavía - de extraer toda la pintura indeleble del sitio parece demasiado descabellado y perjudicial para ser contemplado. $\mathrm{El} \mathrm{CCl}$ ha sugerido incluso que se lleve a cabo la limpieza de tal modo que se produzcan diferencias de contraste entre los grabados y el fondo de roca, en función de la cronología, icompletamente especulativa! de los petroglifos. Esta opción que ha sido admitida alegremente, exigiría de una "aguda y perspicaz opinión", capaz de asegurar que las zonas limpiadas corresponden realmente a los grabados originalmente esculpidos, y más aún cuando la pintura no ha sido aplicada siempre de forma correcta. En otras palabras, el método abrasivo podría casi con toda certeza y de forma irreversible, - a diferencia de la pintura-crear nuevos contornos y por esto cambiar la forma de algunas figuras, sobreponiendo la interpretación del operario de la herramienta de aire sobre la del artista de la pintura indeleble, y en el proceso destruir los últimos vestigios originales de la superficie de los petroglifos.

Este proyecto puede definirse inequívocamente como un vandalismo profesional, capaz de arruinar cualquier esperanza de aplicar en el futuro métodos científicos analíticos. Devolver los petroglifos a su estado prístino, sin pátina, es una opción viable; no obstante si se considera que tal elección es necesaria en Peterborough, ésta debería centrarse en la creación de una reproducción a tamaño natural, y no en la consecucción de un original destruido por dicha actitud.

Para resumir, todas las noticias que hemos tenido a través de diversos artículos - hasta la publicación de nuestro último artículo- han sido autoalabanzas y afirmaciones paliativas por parte de los autores, similares a las repetidas por Isabel García Fernández. Con todo, resulta primordial que otras personas e instituciones que están considerando proyectos similares conozcan de forma precisa qué dificultades se encontraron durante la construcción, y qué defectos de diseño se hallaron, qué daño se produjo durante y después de la construcción, los errores que se realizaron en la creación y mantenimiento del sitio, y las maneras en que el diseño de mantenimiento podría haber sido mejorado.

Tomando como ejemplo Peterborough, u otros sitios similares (como 
Besovy Sledki en Karelia, Rusia) queda claro que la cubrición de lugares con arte rupestre con una estructura artifical altera el medioambiente natural de una forma dramática. Mediante el aislamiento de los procesos naturales que se consideran perjudiciales, también se aisla a las representaciones de los procesos naturales que pudieran contribuir a su preservación. Más aún, se introduce un medioambiente artificial que muy posiblemente podría originar nuevos riesgos para su conservación. No conocemos los efectos a largo plazo de tan importante y masiva intervención medioambiental sobre un arte rupestre que ha sobrevivido centurias o milenios de deterioro natural y ha convivido probablemente en equilibrio con su medioambiente natural. Encerrar este tipo de lugares los relega al «status" de objetos museo y los aisla de su marco natural, alterando sin duda su significado cultural, y convirtiendo los museos en mausoléos.

Un proyecto de estas características no se debería de haber llevado nunca a cabo sin un programa de control y de mantenimiento a largo plazo, que en este caso el $\mathrm{CCl}$ estaría éticamente obligado a proporcionar. En Peterborough, en lugar de dirigir tal programa a un tipo de estudios, que podrían haber sido de inmensa ayuda para el campo de la conservación del arte rupestre, el $\mathrm{CCl}$ se ha mostrado mucho más preocupado por desarrollar pretenciosos planes como extraer la pintura indeleble. En lugar de revelar la extensión completa del daño causado durante y después de la construcción de la estructura, para que los administradores internacionales del arte rupestre puedan aprender del desastre, se propone su avance destructivo. Por tanto, nos mostramos horrorizados ante el limitado y estrecho comentario de Isabel García Fernández: «Hay que destacar la labor del laboratorio de analítica del Instituto Canadiense de Conservación, en la conservación y preservación del arte rupestre, no sólo en Canadá, sino en todo el mundo". La decisión del $\mathrm{CCl}$, de expandir su espíritu y sus métodos a otros países resulta escalofriante para aquellos que estamos interesados en el arte rupestre, su conservación y musealización.

\section{BIBLIOGRAFIA}

Bahn, P.G., Bednarik, R.G. y Steingring, J.: "The Peterborough site: reflections on massive intervention in rock art". Rock Art Research vol. 12(1), 1995, pp. 29-41.

WAINWRIGHT, I.N. et alii: «The rock art of Petroglyphs Provincial Park: conservation and recording". Unpublished report, Canadian Conservation Institute, Ottawa, $72 \mathrm{pp}$.

WAINWRIGHT, I.N. M. y STONE, T.G.: «Experiments to remove wax crayon from petroglyphs in marble». American Indian Rock Art vol. 16, 1990, pp. 21-33. 